\title{
On a Stochastic Lotka-Volterra Competitive System with Distributed Delay and General Lévy Jumps
}

\author{
Lijie Zhang, ${ }^{1}$ Chun Lu, ${ }^{1,2}$ and Hui Liu ${ }^{1}$ \\ ${ }^{1}$ Department of Mathematics, Qingdao University of Technology, Qingdao 266520, China \\ ${ }^{2}$ Department of Mathematics, Harbin Institute of Technology, Weihai 264209, China \\ Correspondence should be addressed to Chun Lu; mathlc@163.com
}

Received 6 August 2016; Accepted 9 November 2016

Academic Editor: Ana Carpio

Copyright (c) 2016 Lijie Zhang et al. This is an open access article distributed under the Creative Commons Attribution License, which permits unrestricted use, distribution, and reproduction in any medium, provided the original work is properly cited.

\begin{abstract}
This paper considers a stochastic competitive system with distributed delay and general Lévy jumps. Almost sufficient and necessary conditions for stability in time average and extinction of each population are established under some assumptions. And two facts are revealed: both stability in time average and extinction have closer relationships with the general Lévy jumps, firstly; and secondly, the distributed delay has no effect on the stability in time average and extinction of the stochastic system. Some simulation figures, which are obtained by the split-step $\theta$-method to discretize the stochastic model, are introduced to support the analytical findings.
\end{abstract}

\section{Introduction}

In recent years, delay differential equations has been used in the study of population dynamics. A famous competitive system with distributed delay can be expressed by

$$
\begin{aligned}
& \frac{d y_{1}(t)}{d t} \\
& \quad=y_{1}(t)\left[b_{1}-a_{11} y_{1}(t)-a_{12} \int_{-\tau_{2}}^{0} y_{2}(t+\theta) d \mu_{2}(\theta)\right], \\
& \frac{d y_{2}(t)}{d t} \\
& =y_{2}(t)\left[b_{2}-a_{21} \int_{-\tau_{1}}^{0} y_{1}(t+\theta) d \mu_{1}(\theta)-a_{22} y_{2}(t)\right],
\end{aligned}
$$

where $y_{i}(t)$ denotes the size of the $i$ th population, $b_{i}, a_{i j}$, and $\tau_{i}$ are all positive constants, and $\mu_{i}$ is a probability measure on $\left[-\tau_{i}, 0\right]$. There is an extensive literature concerned with the dynamics of (1) and we here only mention Kuang and Smith [1], Faria [2], Freedman and Wu [3], Bereketoglu and Györi [4], and Gopalsamy [5] among many others. In particular, Kuang (see [6, p. 231]) claimed that if $\Psi_{1}>0$ and $\Psi_{2}>0$, then model (1) has a positive equilibrium $x^{*}=\left(x_{1}^{*}, x_{2}^{*}\right)=$ $\left(\Psi_{1} / \Psi, \Psi_{2} / \Psi\right)$ which is globally asymptotically stable, where
$\Psi=a_{11} a_{22}-a_{12} a_{21}, \Psi_{1}=b_{1} a_{22}-b_{2} a_{12}$, and $\Psi_{2}=b_{2} a_{11}-b_{1} a_{21}$. It is important to point out that if $\Psi_{1}>0$ and $\Psi_{2}>0$, then $\Psi>0$.

In the real world, the intrinsic growth rates of many species are always disturbed by environmental noises (see, e.g., [7-10]), which was recognized by many scholars in recent years (see, e.g., [11-14]). In particular, May [7] has pointed out that, due to environmental noises, the birth rates, carrying capacity, and other parameters involved in the system should be stochastic. In this paper, we assume that the parameters $b_{1}$ and $b_{2}$ are stochastic; then by the central limit theorem, we can replace $b_{1}$ and $b_{2}$ by

$$
\begin{aligned}
& b_{1} \longrightarrow b_{1}+\sigma_{1} \dot{B}_{1}(t), \\
& b_{2} \longrightarrow b_{2}+\sigma_{2} \dot{B}_{2}(t),
\end{aligned}
$$

where, for $i=1,2, B_{i}(t)$ represents a standard Brownian motion defined on a complete probability space $(\Omega, \mathscr{F}, \mathscr{P})$ and $\sigma_{i}^{2}$ is the intensity of the noise.

On the other hand, the population systems may suffer sudden environmental perturbations, that is, some jump type stochastic perturbations, for example, earthquakes, hurricanes, and epidemics. Some scholars have concentrated on the population systems with compensator jumps, and some significant and interesting results have been obtained (see, 
e.g., [15-19]). Bao et al. $[15,16]$ did pioneering work in this field. In addition, Zou et al. [20-22] introduce a general Lévy jumps, which is more reasonable and complicated than the compensator jumps from the viewpoint of biomathematics (see [20]), into population models for the first time. However, there are no articles introducing the general Lévy jumps into population models with distributed delay, to the best of our knowledge. Motivated by these, we consider the famous stochastic competitive system with distributed delay and general Lévy jumps:

$$
\begin{aligned}
& d y_{1}(t)=y_{1}\left(t^{-}\right) \\
& \cdot\left[b_{1}-a_{11} y_{1}\left(t^{-}\right)-a_{12} \int_{-\tau_{2}}^{0} y_{2}\left(t^{-}+\theta\right) d \mu_{2}(\theta)\right] d t \\
& +\sigma_{1} y_{1}\left(t^{-}\right) d B_{1}(t)+y_{1}\left(t^{-}\right) \int_{\mathbb{Y}} \gamma_{1}(u) N(d t, d u), \\
& d y_{2}(t)=y_{2}\left(t^{-}\right) \\
& \cdot\left[b_{2}-a_{21} \int_{-\tau_{1}}^{0} y_{1}\left(t^{-}+\theta\right) d \mu_{1}(\theta)-a_{22} y_{2}\left(t^{-}\right)\right] d t \\
& +\sigma_{2} y_{2}\left(t^{-}\right) d B_{2}(t)+y_{2}\left(t^{-}\right) \int_{\mathbb{Y}} \gamma_{2}(u) N(d t, d u),
\end{aligned}
$$

where $y_{i}\left(t^{-}\right)=\lim _{s \uparrow t} y_{i}(s), N(d t, d u)$ is a real-valued Poisson counting measure with characteristic measure $\lambda$ on a measurable subset $\mathbb{Y}$ of $\bar{R}_{+}$with $\lambda(\mathbb{Y})<+\infty, \widetilde{N}(d t, d u)=N(d t, d u)-$ $\lambda(d u) d t, \gamma(u)$ is bounded function, and $\gamma(u)>-1, u \in \mathbb{Y}$; furthermore, we assume that $B_{i}(t)$ is independent of $N$. Let the initial data $\xi(t)=\left(\xi_{1}(t), \xi_{2}(t)\right) \in C\left([-\tau, 0], R_{+}^{2}\right)$, where $C\left([-\tau, 0] ; R_{+}^{2}\right)$ represents the family of continuous functions from $[-\tau, 0]$ to $R_{+}^{2}$ with the norm $\left\|\xi_{i}\right\|=\sup _{-\tau \leq t \leq 0}\left|\xi_{i}(t)\right|$, $i=1,2, \tau=\max \left\{\tau_{1}, \tau_{2}\right\}$. Other parameters are defined and required as before.

For convenience, we introduce the following notations:

$$
\begin{aligned}
R_{+}^{2} & =\left\{g=\left(g_{1}, g_{2}\right) \in R^{2} \mid g_{i}>0, i=1,2\right\}, \\
\langle f(t)\rangle & =t^{-1} \int_{0}^{t} f(s) d s, \\
\eta_{i} & =\int_{\mathbb{Y}} \ln \left(\frac{1}{1+\gamma_{i}(u)}\right) \lambda(d u), \quad i=1,2, \\
\widetilde{\Psi}_{1} & =0.5 a_{22} \sigma_{1}^{2}-0.5 a_{12} \sigma_{2}^{2}+a_{22} \eta_{1}-a_{12} \eta_{2}, \\
\widetilde{\Psi}_{2} & =0.5 a_{11} \sigma_{2}^{2}-0.5 a_{21} \sigma_{1}^{2}+a_{11} \eta_{2}-a_{21} \eta_{1} .
\end{aligned}
$$

Moreover, we impose the following assumptions in this paper.

Assumption 1. There exists a positive constant $c$ such that $|\ln (1+\gamma(u))| \leq c$ for $\gamma(u)>-1$.

In this paper, we consider a stochastic competitive system with distributed delay and general Lévy jumps. Unlike the deterministic system, the stochastic system does not have an interior equilibrium. Therefore, we cannot investigate the stability of the stochastic system. In Section 2, we show that the solution to system (3) will tend to a point in time average. Furthermore, we establish almost sufficient and necessary conditions for stability in time average and extinction of each population. In Section 3, we present an example to illustrate our mathematical findings. Section 4 gives the conclusions and future directions of the research.

\section{Main Content}

Lemma 2 (see Liu et al. [23]). Suppose that $z(t) \in C(\Omega \times$ $\left.[0,+\infty), R_{+}\right)$.

(i) If there exist two positive constants $T$ and $\rho_{0}$ such that $\ln z(t) \leq \rho t-\rho_{0} \int_{0}^{t} z(s) d s+\sum_{i=1}^{2} \alpha_{i} B_{i}(t)$ for all $t \geq T$, where $\alpha_{i}, i=1,2$, are constants, then

$$
\begin{gathered}
\lim \sup _{t \rightarrow+\infty}\langle z(t)\rangle \leq \frac{\rho}{\rho_{0}} \quad \text { a.s., if } \rho \geq 0 ; \\
\lim _{t \rightarrow+\infty} z(t)=0 \quad \text { a.s., if } \rho<0 .
\end{gathered}
$$

(ii) If there exist three positive constants $T, \rho$, and $\rho_{0}$ such that $\ln z(t) \geq \lambda t-\rho_{0} \int_{0}^{t} z(s) d s+\sum_{i=1}^{2} \alpha_{i} B_{i}(t)$ for all $t \geq T$, then $\lim \inf _{t \rightarrow+\infty}\langle z(t)\rangle \geq \rho / \rho_{0}$ a.s.

In order for the model to be significant, we shall show that the solution is global and nonnegative. However, theorem of existence and uniqueness ([24-28]) is not satisfied in system (3). By using method established by Mao et al. [8], we will show existence and uniqueness of the global positive solution of system (3).

Lemma 3. Let Assumption 1 hold. For any given initial value $\xi(t)=\left(\xi_{1}(t), \xi_{2}(t)\right) \in C\left([-\tau, 0], R_{+}^{2}\right)$; then system (3) has a unique positive solution $x(t)=\left(y_{1}(t), y_{2}(t)\right)$ on $t \geq-\tau$ a.s. and the solution satisfies

$$
\limsup _{t \rightarrow+\infty} \frac{\ln y_{i}(t)}{\ln t} \leq 1 \quad \text { a.s., } i=1,2 .
$$

Proof. The proof is similar to Han et al. [29] by defining

$$
V(x)=V_{1}(x)+V_{2}(x),
$$

where

$$
\begin{aligned}
& V_{1}(x)=\sqrt{x_{1}}-1-0.5 \ln x_{1}, \\
& V_{2}(x)=\sqrt{x_{2}}-1-0.5 \ln x_{2} .
\end{aligned}
$$

In addition, applying the inequality, for $i=1,2$,

$$
\begin{aligned}
& \int_{0}^{t} \int_{-\tau}^{0} x_{i}^{2}\left((s+\theta)^{-}\right) d \mu(\theta) d s \\
& \quad=\int_{-\tau}^{0} d \mu(\theta) \int_{\theta}^{t} x_{i}^{2}\left(s^{-}\right) d s \\
& \quad \leq \int_{-\tau}^{0} d \mu(\theta) \int_{-\tau}^{t} x_{i}^{2}\left(s^{-}\right) d s \\
& \quad \leq \int_{-\tau}^{0} \xi_{i}^{2}\left(s^{-}\right) d s+\int_{0}^{t} x_{i}^{2}\left(s^{-}\right) d s .
\end{aligned}
$$


So we omit it here. Now let us prove inequality (6).

Case $1(i=1)$. For any $t \geq 0$, applying the generalized Itô's formula [30] to (3) results in

$$
\begin{aligned}
& d\left(e^{t} \ln y_{1}\right)=e^{t} \ln y_{1} d t+e^{t} d \ln y_{1}=e^{t}\left[\ln y_{1}+b_{1}\right. \\
& -0.5 \sigma_{1}^{2}+\int_{\mathbb{Y}} \ln \left(1+\gamma_{1}(u)\right) \lambda(d u)-a_{11} y_{1} \\
& \left.-a_{12} \int_{-\tau_{2}}^{0} y_{2}\left(t^{-}+\theta\right) d \mu_{2}(\theta)\right] d t+\sigma_{1} d B_{1}(t) \\
& +\int_{\mathbb{Y}} \ln \left(1+\gamma_{1}(u)\right) \widetilde{N}(d t, d u) \leq e^{t}\left[\ln y_{1}+b_{1}\right. \\
& \left.-0.5 \sigma_{1}^{2}+\int_{\mathbb{V}} \ln \left(1+\gamma_{1}(u)\right) \lambda(d u)-a_{11} y_{1}\right] d t \\
& +e^{t} \sigma_{1} d B_{1}(t)+e^{t} \int_{\mathbb{V}} \ln \left(1+\gamma_{1}(u)\right) \widetilde{N}(d t, d u) .
\end{aligned}
$$

Thus

$$
\begin{aligned}
& e^{t} \ln y_{1}(t) \leq \ln y_{1}(0)+\int_{0}^{t} e^{s}\left[\ln y_{1}(s)+b_{1}-0.5 \sigma_{1}^{2}\right. \\
& \left.+\int_{\mathbb{V}} \ln \left(1+\gamma_{1}(u)\right) \lambda(d u)-a_{11} y_{1}(s)\right] d s \\
& +\int_{0}^{t} e^{s} \sigma_{1} d B_{1}(s) \\
& +\int_{0}^{t} e^{s} \int_{\mathbb{V}} \ln \left(1+\gamma_{1}(u)\right) \widetilde{N}(d s, d u) .
\end{aligned}
$$

The rest of proof is analogous with Lemma 4.4 in [15]; we omitted it here.

Case $2(i=2)$. The proof is similar to Case 1 ; we left out it here. The proof is complete.

Theorem 4. For system (3), we suppose that Assumption 1, $\Psi_{1}>0$ and $\Psi_{2}>0$, holds.

(I) If $b_{1}<0.5 \sigma_{1}^{2}+\eta_{1}$ and $b_{2}<0.5 \sigma_{2}^{2}+\eta_{2}$, then both $y_{1}$ and $y_{2}$ are extinctive almost surely (a.s.); that is, $\lim _{t \rightarrow+\infty} y_{i}(t)=0$ a.s., $i=1,2$.

(II) If $b_{1}>0.5 \sigma_{1}^{2}+\eta_{1}$ and $b_{2}<0.5 \sigma_{2}^{2}+\eta_{2}$, then $y_{2}$ is extinctive a.s. and $y_{1}$ is stable in time average a.s.; that is,

$$
\lim _{t \rightarrow+\infty} t^{-1} \int_{0}^{t} y_{1}(s) d s=\frac{\left[b_{1}-0.5 \sigma_{1}^{2}-\eta_{1}\right]}{a_{11}}, \text { a.s.. }
$$

(III) If $b_{1}<0.5 \sigma_{1}^{2}+\eta_{1}$ and $b_{2}>0.5 \sigma_{2}^{2}+\eta_{2}$, then $y_{1}$ is extinctive a.s. and $y_{2}$ is stable in time average a.s.; that is,

$$
\lim _{t \rightarrow+\infty} t^{-1} \int_{0}^{t} y_{2}(s) d s=\frac{\left[b_{2}-0.5 \sigma_{2}^{2}-\eta_{2}\right]}{a_{22}}, \text { a.s.. }
$$

(IV) If $b_{1}>0.5 \sigma_{1}^{2}+\eta_{1}, b_{2}>0.5 \sigma_{2}^{2}+\eta_{2}$

(A) If $\Psi_{1}>\widetilde{\Psi}_{1}$ and $\Psi_{2}<\widetilde{\Psi}_{2}$, then $y_{2}$ is extinctive a.s. and $y_{1}$ is stable in time average a.s.:

$$
\lim _{t \rightarrow+\infty} t^{-1} \int_{0}^{t} y_{1}(s) d s=\frac{\left[b_{1}-0.5 \sigma_{1}^{2}-\eta_{1}\right]}{a_{11}}, \quad \text { a.s. }
$$

(B) If $\Psi_{1}<\widetilde{\Psi}_{1}$ and $\Psi_{2}>\widetilde{\Psi}_{2}$, then $y_{1}$ is extinctive a.s. and $y_{2}$ is stable in time average a.s.:

$$
\lim _{t \rightarrow+\infty} t^{-1} \int_{0}^{t} y_{2}(s) d s=\frac{\left[b_{2}-0.5 \sigma_{2}^{2}-\eta_{2}\right]}{a_{22}}, \quad \text { a.s.; }
$$

(C) If $\Psi_{1}>\widetilde{\Psi}_{1}$ and $\Psi_{2}>\widetilde{\Psi}_{2}$, then both $y_{1}$ and $y_{2}$ are stable in time average a.s.:

$$
\lim _{t \rightarrow+\infty} t^{-1} \int_{0}^{t} y_{1}(s) d s=\frac{\Psi_{1}-\widetilde{\Psi}_{1}}{\Psi}
$$$$
\lim _{t \rightarrow+\infty} t^{-1} \int_{0}^{t} y_{2}(s) d s=\frac{\Psi_{2}-\widetilde{\Psi}_{2}}{\Psi}, \quad \text { a.s. }
$$

Proof. Applying Itô's formula [30] to the first equality in (3), we get

$$
\begin{aligned}
& \ln y_{1}(t)-\ln y_{1}(0) \\
& =\left[b_{1}-0.5 \sigma_{1}^{2}-\eta_{1}\right] t-a_{11} \int_{0}^{t} y_{1}(s) d s \\
& \quad-a_{12} \int_{0}^{t} \int_{-\tau_{2}}^{0} y_{2}(s+\theta) d \mu_{2}(\theta) d s+\sigma_{1} B_{1}(t) \\
& \quad+\int_{0}^{t} \int_{\mathbb{V}} \ln \left(1+\gamma_{1}(u)\right) \widetilde{N}(d s, d u) .
\end{aligned}
$$

Making use of the Fubini theorem and a substitution technique, we have

$$
\begin{aligned}
& \int_{0}^{t} \int_{-\tau_{2}}^{0} y_{2}(s+\theta) d \mu_{2}(\theta) d s=\int_{-\tau_{2}}^{0} d \mu_{2}(\theta) \\
& \cdot \int_{0}^{t} y_{2}(s+\theta) d s=\int_{-\tau_{2}}^{0} d \mu_{2}(\theta) \int_{\theta}^{t+\theta} y_{2}(s) d s \\
& \quad=\int_{-\tau_{2}}^{0} d \mu_{2}(\theta) \\
& \cdot\left[\int_{\theta}^{0} y_{2}(s) d s+\int_{0}^{t} y_{2}(s) d s+\int_{t}^{t+\theta} y_{2}(s) d s\right] \\
& \quad=\int_{-\tau_{2}}^{0} d \mu_{2}(\theta) \int_{\theta}^{0} y_{2}(s) d s+\int_{0}^{t} y_{2}(s) d s \\
& \quad+\int_{-\tau_{2}}^{0} d \mu_{2}(\theta) \int_{t}^{t+\theta} y_{2}(s) d s .
\end{aligned}
$$


Therefore, we derive

$$
\begin{aligned}
& \ln y_{1}(t)-\ln y_{1}(0) \\
& =\left[b_{1}-0.5 \sigma_{1}^{2}-\eta_{1}\right] t-a_{11} \int_{0}^{t} y_{1}(s) d s \\
& \quad-a_{12} \int_{0}^{t} y_{2}(s) d s-a_{12} \int_{-\tau_{2}}^{0} d \mu_{2}(\theta) \int_{\theta}^{0} y_{2}(s) d s \\
& \quad-a_{12} \int_{-\tau_{2}}^{0} d \mu_{2}(\theta) \int_{t}^{t+\theta} y_{2}(s) d s+\sigma_{1} B_{1}(t) \\
& \quad+\int_{0}^{t} \int_{\mathbb{Y}} \ln \left(1+\gamma_{1}(u)\right) \widetilde{N}(d s, d u) .
\end{aligned}
$$

Similarly,

$$
\begin{aligned}
& \ln y_{2}(t)-\ln y_{2}(0) \\
& =\left(b_{2}-0.5 \sigma_{2}^{2}-\eta_{2}\right) t-a_{22} \int_{0}^{t} y_{2}(s) d s \\
& \quad-a_{21} \int_{0}^{t} y_{1}(s) d s-a_{21} \int_{-\tau_{1}}^{0} d \mu_{1}(\theta) \int_{\theta}^{0} y_{1}(s) d s \\
& \quad-a_{21} \int_{-\tau_{1}}^{0} d \mu_{1}(\theta) \int_{t}^{t+\theta} y_{1}(s) d s+\sigma_{2} B_{2}(t) \\
& \quad+\int_{0}^{t} \int_{\mathbb{Y}} \ln \left(1+\gamma_{2}(u)\right) \widetilde{N}(d s, d u) .
\end{aligned}
$$

(I) Assume that $b_{1}<0.5 \sigma_{1}^{2}+\eta_{1}$ and $b_{2}<0.5 \sigma_{2}^{2}+\eta_{2}$. From (9),

$$
\begin{aligned}
t^{-1} \ln \frac{y_{1}(t)}{y_{1}(0)} \leq & b_{1}-0.5 \sigma_{1}^{2}-\eta_{1}+t^{-1} \sigma_{1} B_{1}(t) \\
& +t^{-1} \int_{0}^{t} \int_{\mathbb{V}} \ln \left(1+\gamma_{1}(u)\right) \widetilde{N}(d s, d u) .
\end{aligned}
$$

By the strong law of large numbers for martingales, we therefore have $\lim _{t \rightarrow+\infty} B_{i}(t) / t=0$ a.s., $i=1,2$; meanwhile, we define, for $t \geq 0, M_{i}(t)=\int_{0}^{t} \int_{\mathbb{Y}} \ln \left(1+\gamma_{i}(u)\right) \widetilde{N}(d s, d u)$, $i=1,2$. Under Assumption $1,\left\langle M_{i}\right\rangle(t)=\int_{0}^{t} \int_{\mathbb{V}}(\ln (1+$ $\left.\gamma_{i}(u)\right)^{2} \lambda(d u) d s \leq c^{2} t \lambda(\mathbb{Y})$, making use of the strong law of large numbers for local martingales (see, e.g., [31]), we then derive

$$
\lim _{t \rightarrow+\infty} \frac{1}{t} \int_{0}^{t} \int_{\mathbb{Y}} \ln \left(1+\gamma_{i}(u)\right) \widetilde{N}(d s, d u)=0 \quad \text { a.s., }
$$

and $b_{1}<0.5 \sigma_{1}^{2}+\eta_{1}$ that

$$
\limsup _{t \rightarrow+\infty} t^{-1} \ln y_{1}(t) \leq b_{1}-0.5 \sigma_{1}^{2}-\eta_{1}<0
$$

Consequently, $\lim _{t \rightarrow+\infty} y_{1}(t)=0$, a.s. Similarly, by (19), we can show that if $b_{2}<0.5 \sigma_{2}^{2}+\eta_{2}$, then $\lim _{t \rightarrow+\infty} y_{2}(t)=0$, a.s.

(II) Suppose that $b_{1}>0.5 \sigma_{1}^{2}+\eta_{1}$ and $b_{2}<0.5 \sigma_{2}^{2}+\eta_{2}$. Since $b_{2}<0.5 \sigma_{2}^{2}+\eta_{2}$, then, by $(\mathrm{I}), \lim _{t \rightarrow+\infty} y_{2}(t)=0$, a.s. Hence, making use of (9), for arbitrary $\varepsilon>0$, there is $T>0$ such that, for $t \geq T$,

$$
\begin{aligned}
& \frac{-\varepsilon}{2} \leq a_{12} t^{-1} \int_{0}^{t} \int_{-\tau_{2}}^{0} y_{2}(s+\theta) d \mu_{2}(\theta) d s \leq \frac{\varepsilon}{2}, \\
& \frac{-\varepsilon}{2} \leq t^{-1} \ln y_{1}(0) \leq \frac{\varepsilon}{2} .
\end{aligned}
$$

Substituting the above inequalities into (19), we can see that, for $t \geq T$,

$$
\begin{aligned}
\ln y_{1}(t) \leq & \left(b_{1}-0.5 \sigma_{1}^{2}-\eta_{1}+\varepsilon\right) t-a_{11} \int_{0}^{t} y_{1}(s) d s \\
& +\sigma_{1} B_{1}(t) \\
& +\int_{0}^{t} \int_{\mathbb{Y}} \ln \left(1+\gamma_{1}(u)\right) \widetilde{N}(d s, d u), \\
\ln y_{1}(t) \geq & \left(b_{1}-0.5 \sigma_{1}^{2}-\eta_{1}-\varepsilon\right) t-a_{11} \int_{0}^{t} y_{1}(s) d s \\
& +\sigma_{1} B_{1}(t) \\
& +\int_{0}^{t} \int_{\mathbb{Y}} \ln \left(1+\gamma_{1}(u)\right) \widetilde{N}(d s, d u) .
\end{aligned}
$$

Since $b_{1}>0.5 \sigma_{1}^{2}+\eta_{1}$, we can choose $\varepsilon$ sufficiently small such that $b_{1}-0.5 \sigma_{1}^{2}-\eta_{1}-\varepsilon>0$. Applying (i) and (ii) in Lemma 2 to (25) and (26), respectively, we derive

$$
\begin{aligned}
\frac{b_{1}-0.5 \sigma_{1}^{2}-\eta_{1}-\varepsilon}{a_{11}} & \leq \liminf _{t \rightarrow+\infty}\left\langle y_{1}(t)\right\rangle \\
& \leq \limsup _{t \rightarrow+\infty}\left\langle y_{1}(t)\right\rangle \\
& \leq \frac{b_{1}-0.5 \sigma_{1}^{2}-\eta_{1}+\varepsilon}{a_{11}}, \text { a.s. }
\end{aligned}
$$

Let $\varepsilon \rightarrow 0$. Then, we have $\lim _{t \rightarrow+\infty}\left\langle y_{1}(t)\right\rangle=\left[b_{1}-0.5 \sigma_{1}^{2}-\right.$ $\left.\eta_{1}\right] / a_{11}$, a.s.

The proof of (III) is homogeneous with (II) by symmetry; hence it is omitted.

Now let us prove (IV). For $i=1,2$, consider the following equation:

$$
\begin{aligned}
d z_{i}(t)= & z_{i}(t)\left[b_{i}-a_{i i} z_{i}(t)\right] d t+\sigma_{i} z_{i}(t) d B_{i}(t) \\
& +z_{1}\left(t^{-}\right) \int_{\mathbb{Y}} \gamma_{i}(u) N(d t, d u), \\
z_{i}(\theta)= & y_{i}(\theta), \quad \theta \in[-\tau, 0] .
\end{aligned}
$$

In virtue of the classic stochastic comparison theorem [32], we can find that

$$
\begin{aligned}
& y_{1}(t) \leq z_{1}(t), \\
& y_{2}(t) \leq z_{2}(t) .
\end{aligned}
$$


Since $b_{i}>0.5 \sigma_{i}^{2}+\eta_{i}, i=1,2$, similar to the proof of (II), we can show that

$$
\begin{aligned}
& \lim _{t \rightarrow+\infty}\left\langle z_{i}(t)\right\rangle=\lim _{t \rightarrow+\infty} t^{-1} \int_{0}^{t} z_{i}(s) d s \\
&=\frac{\left(b_{i}-0.5 \sigma_{i}^{2}-\eta_{2}\right)}{a_{i i}}, \quad \text { a.s., } i=1,2, \\
& \lim _{t \rightarrow+\infty}\left\langle z_{i}(t)\right\rangle=\lim _{t \rightarrow+\infty} t^{-1} \int_{0}^{t+\theta} z_{i}(s) d s \\
&=\frac{\left(b_{i}-0.5 \sigma_{i}^{2}-\eta_{2}\right)}{a_{i i}}, \\
& \text { a.s., }-\tau \leq \theta \leq 0, i=1,2 .
\end{aligned}
$$

Thus

$$
\begin{aligned}
& \lim _{t \rightarrow+\infty} t^{-1} \int_{t+\theta}^{t} z_{1}(s) d s \\
& =\lim _{t \rightarrow+\infty}\left(t^{-1} \int_{0}^{t} z_{1}(s) d s-t^{-1} \int_{0}^{t+\theta} z_{1}(s) d s\right)=0 \\
& \lim _{t \rightarrow+\infty} t^{-1} \int_{t+\theta}^{t} z_{2}(s) d s=0, \quad \text { a.s., }
\end{aligned}
$$

which, together with (29), implies that

$$
\begin{aligned}
& \lim _{t \rightarrow+\infty} t^{-1} \int_{t+\theta}^{t} y_{1}(s) d s=0, \\
& \lim _{t \rightarrow+\infty} t^{-1} \int_{t+\theta}^{t} y_{2}(s) d s=0, \quad \text { a.s. }
\end{aligned}
$$

On the other hand, calculating $(2.5) \times a_{11}-(2.4) \times a_{21}$ deduces

$$
\begin{aligned}
a_{11} \ln \frac{y_{2}(t)}{y_{2}(0)}= & -a_{11} a_{21} \int_{-\tau_{1}}^{0} d \mu_{1}(\theta) \int_{\theta}^{0} y_{1}(s) d s \\
& -a_{11} a_{21} \int_{-\tau_{1}}^{0} d \mu_{1}(\theta) \int_{t}^{t+\theta} y_{1}(s) d s \\
& +a_{21} a_{12} \int_{-\tau_{2}}^{0} d \mu_{2}(\theta) \int_{\theta}^{0} y_{2}(s) d s \\
& +a_{21} a_{12} \int_{-\tau_{2}}^{0} d \mu_{2}(\theta) \int_{t}^{t+\theta} y_{2}(s) d s \\
& +a_{21} \ln \frac{y_{1}(t)}{y_{1}(0)}+\left(\Psi_{2}-\widetilde{\Psi}_{2}\right) t \\
& -\Psi \int_{0}^{t} y_{2}(s) d s-a_{21} \sigma_{1} B_{1}(t) \\
& -a_{21} \int_{0}^{t} \int_{\mathbb{Y}} \ln \left(1+\gamma_{1}(u)\right) \widetilde{N}(d s, d u) \\
& +a_{11} \sigma_{2} B_{2}(t) \\
& +a_{11} \int_{0}^{t} \int_{\mathbb{V}} \ln \left(1+\gamma_{2}(u)\right) \widetilde{N}(d s, d u) .
\end{aligned}
$$

By virtue of (6) and (32), for arbitrary $\varepsilon>0$, there is $T>0$ such that, for $t \geq T$,

$$
\begin{aligned}
& t^{-1} a_{21} \ln \frac{y_{1}(t)}{y_{1}(0)}<\frac{\varepsilon}{3} \\
& t^{-1} a_{11} \ln y_{2}(0)<\frac{\varepsilon}{3} \\
& -a_{11} a_{21} t^{-1} \int_{-\tau_{1}}^{0} d \mu_{1}(\theta) \int_{\theta}^{0} y_{1}(s) d s \\
& -a_{11} a_{21} t^{-1} \int_{-\tau_{1}}^{0} d \mu_{1}(\theta) \int_{t}^{t+\theta} y_{1}(s) d s \\
& +a_{21} a_{12} t^{-1} \int_{-\tau_{2}}^{0} d \mu_{2}(\theta) \int_{\theta}^{0} y_{2}(s) d s \\
& +a_{21} a_{12} t^{-1} \int_{-\tau_{2}}^{0} d \mu_{2}(\theta) \int_{t}^{t+\theta} y_{2}(s) d s \leq \frac{\varepsilon}{3} .
\end{aligned}
$$

Substituting the above inequalities into (33) results in

$$
\begin{aligned}
a_{11} \ln y_{2}(t) \leq & \left(\Psi_{2}-\widetilde{\Psi}_{2}+\varepsilon\right) t-\Psi \int_{0}^{t} y_{2}(s) d s \\
& -a_{21} \sigma_{1} B_{1}(t)+a_{11} \sigma_{2} B_{2} \\
& +a_{11} \int_{0}^{t} \int_{\mathbb{V}} \ln \left(1+\gamma_{2}(u)\right) \widetilde{N}(d s, d u),
\end{aligned}
$$

for $t>T$. Meanwhile, calculating $(2.4) \times a_{22}-(2.5) \times a_{12}$ yields

$$
\begin{aligned}
a_{22} \ln \frac{y_{1}(t)}{y_{1}(0)}= & -a_{11} a_{21} \int_{-\tau_{1}}^{0} d \mu_{1}(\theta) \int_{\theta}^{0} y_{1}(s) d s \\
& -a_{11} a_{21} \int_{-\tau_{1}}^{0} d \mu_{1}(\theta) \int_{t}^{t+\theta} y_{1}(s) d s \\
& +a_{12} a_{21} \int_{-\tau_{1}}^{0} d \mu_{1}(\theta) \int_{\theta}^{0} y_{1}(s) d s \\
& -a_{12} a_{21} \int_{-\tau_{1}}^{0} d \mu_{1}(\theta) \int_{t}^{t+\theta} y_{1}(s) d s \\
& +a_{12} \ln \frac{y_{2}(t)}{y_{2}(0)}+\left(\Psi_{1}-\widetilde{\Psi}{ }_{1}\right) t \\
& -\Psi \int_{0}^{t} y_{1}(s) d s+a_{22} \sigma_{1} B_{1}(t) \\
& +a_{22} \int_{0}^{t} \int_{\mathbb{Y}} \ln \left(1+\gamma_{1}(u)\right) \widetilde{N}(d s, d u) \\
& -a_{12} \sigma_{2} B_{2}(t) \\
& -a_{12} \int_{0}^{t} \int_{\mathbb{Y}} \ln \left(1+\gamma_{2}(u)\right) \widetilde{N}(d s, d u) .
\end{aligned}
$$


Using the same way, by (36) we can have that, for $t>T$,

$$
\begin{aligned}
a_{22} \ln y_{1}(t) \leq & \left(\Psi_{1}-\widetilde{\Psi}_{1}+\varepsilon\right) t-\Psi \int_{0}^{t} y_{1}(s) d s \\
& +a_{22} \sigma_{1} B_{1}(t) \\
& +a_{22} \int_{0}^{t} \int_{\mathbb{Y}} \ln \left(1+\gamma_{1}(u)\right) \widetilde{N}(d s, d u) \\
& -a_{12} \sigma_{2} B_{2}(t) \\
& -a_{12} \int_{0}^{t} \int_{\mathbb{Y}} \ln \left(1+\gamma_{2}(u)\right) \widetilde{N}(d s, d u) .
\end{aligned}
$$

(A) Suppose $\Psi_{1}>\widetilde{\Psi}_{1}$ and $\Psi_{2}<\widetilde{\Psi}_{2}$. Note that $\Psi_{2}<\widetilde{\Psi}_{2}$, and then let $\varepsilon$ be sufficiently small such that $\Psi_{2}-\widetilde{\Psi}_{2}+\varepsilon<0$. Applying (i) in Lemma 2 to (35) gives $\lim _{t \rightarrow+\infty} y_{2}(t)=0$, a.s. The proof of $\lim _{t \rightarrow+\infty}\left\langle y_{1}(t)\right\rangle=\left(b_{1}-0.5 \sigma_{1}^{2}-\eta_{1}\right) / a_{11}$, a.s., is similar to (II) and hence is omitted.

The proof of (B) is similar to (A) by symmetry and hence is left out.

(C) Suppose that $\Psi_{1}>\widetilde{\Psi}_{1}$ and $\Psi_{2}>\widetilde{\Psi}_{2}$. Since $\Psi_{2}>\widetilde{\Psi}_{2}$, it then follows from (33) and Lemma 2 that

$$
\limsup _{t \rightarrow+\infty}\left\langle y_{2}(t)\right\rangle \leq \frac{\Psi_{2}-\widetilde{\Psi}_{2}+\varepsilon}{\Psi}, \quad \text { a.s. }
$$

Making use of the arbitrariness of $\varepsilon$, we can see that

$$
\limsup _{t \rightarrow+\infty}\left\langle y_{2}(t)\right\rangle \leq \frac{\Psi_{2}-\widetilde{\Psi}_{2}}{\Psi}, \text { a.s. }
$$

It follows from (37), Lemma 2, and the arbitrariness of $\varepsilon$ that

$$
\limsup _{t \rightarrow+\infty}\left\langle y_{1}(t)\right\rangle \leq \frac{\Psi_{1}-\widetilde{\Psi}_{1}}{\Psi}, \quad \text { a.s. }
$$

likewise. Let $\varepsilon$ be sufficiently small such that $a_{11}\left(\left(\Psi_{2}-\right.\right.$ $\left.\left.\widetilde{\Psi}_{2}\right) / \Psi\right)-\varepsilon>0$. When (32) and (39) are used in (19), we get

$$
\begin{aligned}
& t^{-1} \ln y_{1}(t)=t^{-1} \ln y_{1}(0)+b_{1}-0.5 \sigma_{1}^{2}-\eta_{1} \\
& -a_{11}\left\langle y_{1}(t)\right\rangle-a_{12}\left\langle y_{2}(t)\right\rangle+\frac{\sigma_{1} B_{1}(t)}{t} \\
& +t^{-1} \int_{0}^{t} \int_{\mathbb{Y}} \ln \left(1+\gamma_{1}(u)\right) \widetilde{N}(d s, d u) \\
& -a_{12} t^{-1}\left[\int_{-\tau_{2}}^{0} d \mu_{2}(\theta) \int_{\theta}^{0} y_{2}(s) d s\right. \\
& \left.+\int_{-\tau_{2}}^{0} d \mu_{2}(\theta) \int_{t}^{t+\theta} y_{2}(s) d s\right] \geq b_{1}-0.5 \sigma_{1}^{2}-\eta_{1}-\varepsilon \\
& -a_{11}\left\langle y_{1}(t)\right\rangle-a_{12} \limsup _{t \rightarrow+\infty}\left\langle y_{2}(t)\right\rangle+\frac{\sigma_{1} B_{1}(t)}{t}
\end{aligned}
$$

$$
\begin{aligned}
& +t^{-1} \int_{0}^{t} \int_{\mathbb{Y}} \ln \left(1+\gamma_{1}(u)\right) \widetilde{N}(d s, d u) \geq b_{1}-0.5 \sigma_{1}^{2} \\
& -\eta_{1}-\varepsilon-a_{11}\left\langle y_{1}(t)\right\rangle-a_{12} \frac{\Psi_{2}-\widetilde{\Psi}_{2}}{\Psi}+\frac{\sigma_{1} B_{1}(t)}{t} \\
& +t^{-1} \int_{0}^{t} \int_{\mathbb{V}} \ln \left(1+\gamma_{1}(u)\right) \widetilde{N}(d s, d u)=a_{11} \\
& \cdot \frac{\Psi_{1}-\widetilde{\Psi}_{1}}{\Psi}-\varepsilon-a_{11}\left\langle y_{1}(t)\right\rangle+\frac{\sigma_{1} B_{1}(t)}{t} \\
& +t^{-1} \int_{0}^{t} \int_{\mathbb{Y}} \ln \left(1+\gamma_{1}(u)\right) \widetilde{N}(d s, d u)
\end{aligned}
$$

for sufficiently large $t$. In virtue of (ii) in Lemma 2 and the arbitrariness of $\varepsilon$, we get

$$
\liminf _{t \rightarrow+\infty}\left\langle y_{1}(t)\right\rangle \geq \frac{\Psi_{1}-\widetilde{\Psi}_{1}}{\Psi}, \quad \text { a.s. }
$$

Similarly, substituting (32) and (40) into (20) brings about $\lim _{t \rightarrow+\infty} \inf \left\langle y_{2}(t)\right\rangle \geq\left(\Psi_{2}-\widetilde{\Psi}_{2}\right) / \Psi$, a.s. This, together with (39), (40), and (42), means $\lim _{t \rightarrow+\infty}\left\langle y_{1}(t)\right\rangle=\left(\Psi_{1}-\widetilde{\Psi}_{1}\right) / \Psi$ and $\lim _{t \rightarrow+\infty}\left\langle y_{2}(t)\right\rangle=\left(\Psi_{2}-\widetilde{\Psi}_{2}\right) / \Psi$, a.s.

Remark 5. It is important to designate that if $b_{1}>0.5 \sigma_{1}^{2}+\eta_{1}$, $b_{2}>0.5 \sigma_{2}^{2}+\eta_{2}$, and $\Psi>0$, then $\Psi_{1}<\widetilde{\Psi}_{1}$ and $\Psi_{2}<\widetilde{\Psi}_{2}$ cannot hold simultaneously.

Remark 6. Theorem 4 implies an important fact that when $-1<\gamma_{i}(u)<0, i=1,2$, the jump process can result in extinction of the population $y_{i}(t)$, for example, earthquakes and hurricanes, and when $\gamma_{i}(u)>0, i=1,2$, the jump process is always advantage for the population $y_{i}(t)$, for example, ocean red tide.

Remark 7. From the perspective of the condition in Theorem 4, the distributed delay does not influence some the properties including extinction and stability in time average.

\section{Numerical Simulations}

In this section, we employ the split-step $\theta$-method, whose approximate solution is mean-square convergent with order $p=0.5$ (see $[32,33])$, to discretize (3). Here, we choose the initial data $\xi(t)=\left(0.3 e^{t}, 0.4 e^{t}\right), b_{1}=0.59, b_{2}=0.51$, $a_{11}=0.8, a_{12}=0.39, a_{21}=0.51, a_{22}=0.69, \tau_{1}=\tau_{2}=0.3$, $\mathbb{Y}=(0,+\infty)$, and $\lambda(\mathbb{Y})=1$. Then $\Psi=0.36$. The main difference between the conditions of the following Case 1Case 4 is that the values of $\gamma_{1}(u)$ and $\gamma_{2}(u)$ are different. In Case 1 , we let $\gamma_{1}(u)=\gamma_{2}(u)=0$ and $\sigma_{1}^{2}=\sigma_{2}^{2}=0$. Then by virtue of Kuangs work [6], we have that the positive equilibrium $x^{*}=\left(\Psi_{1} / \Psi, \Psi_{2} / \Psi\right)=(0.612,0.277)$ is globally asymptotically stable. Figure $1(\mathrm{a})$ verifies this. In Case 2, we set $\gamma_{1}(u)=-0.1, \gamma_{2}(u)=-0.2$, and $\sigma_{1}^{2}=0.2, \sigma_{2}^{2}=0.4$. Then 

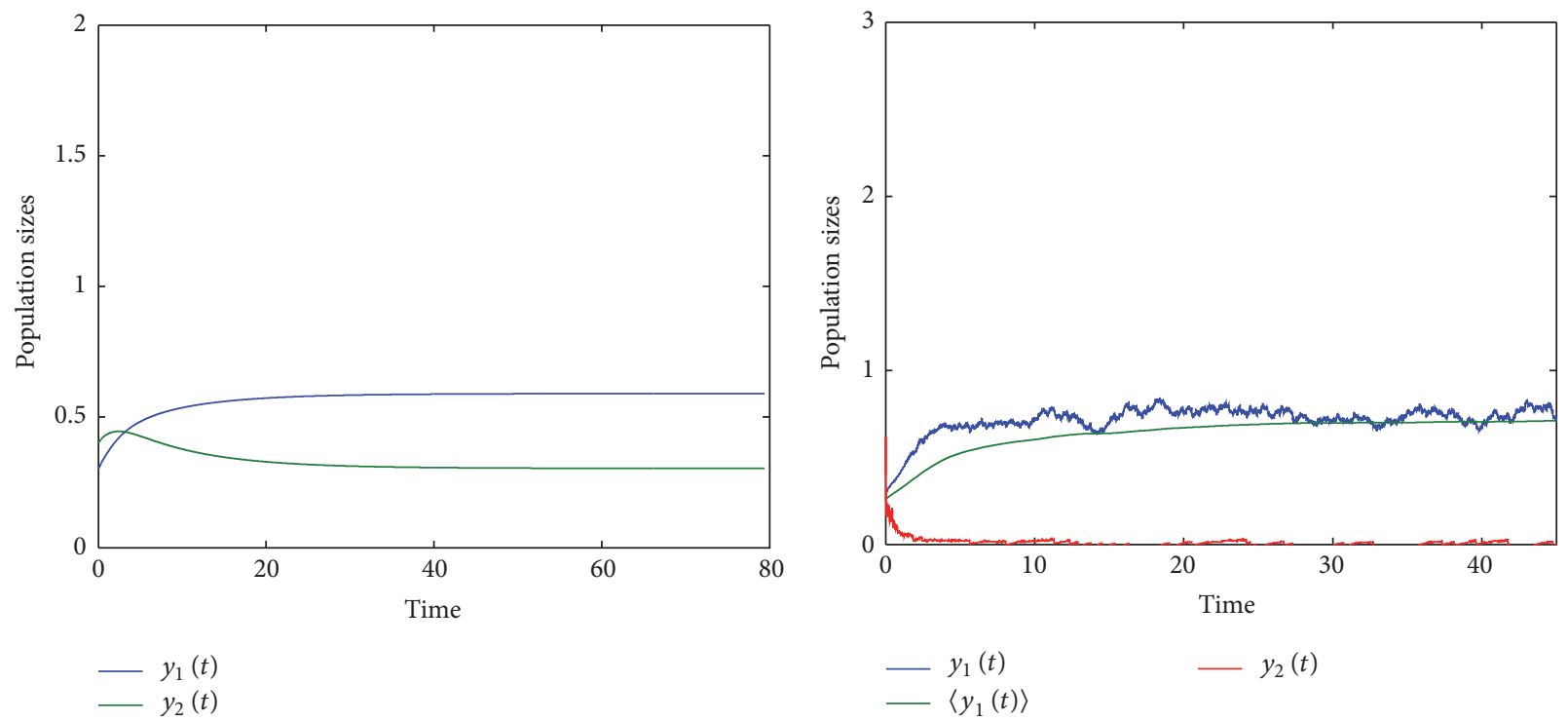

(a)

(b)

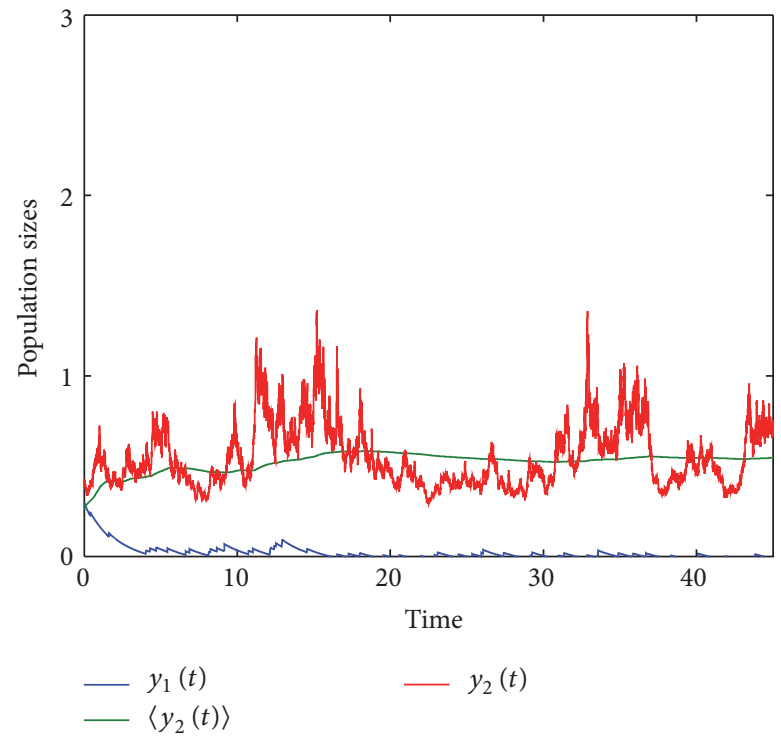

(c)

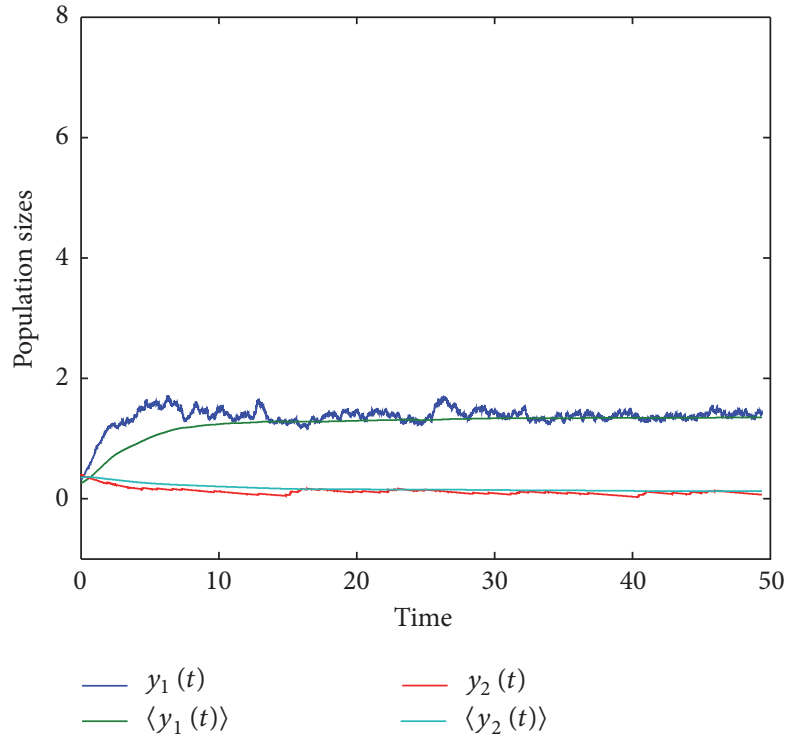

(d)

FIgURE 1: The horizontal axis and the vertical axis in this and following figures represent the time $t$ and the populations size (step size $\Delta t=0.001)$

$\Psi_{1}>\widetilde{\Psi}_{1}=-0.03216$ and $\Psi_{2}=0.1<\widetilde{\Psi}_{2}=0.2386$. In view of (A) in Theorem $4, y_{2}$ goes to extinction and

$$
\begin{aligned}
\lim _{t \rightarrow+\infty} t^{-1} \int_{0}^{t} y_{1}(s) d s & =\frac{b_{1}-0.5 \sigma_{1}^{2}-\eta_{1}}{a_{11}}=\frac{0.595}{0.8} \\
& =0.74 .
\end{aligned}
$$

Figure 1(b) confirms this. In Case 3, we choose $\gamma_{1}(u)=-0.8$, $\gamma_{2}(u)=0.01, \sigma_{1}^{2}=0.2$, and $\sigma_{2}^{2}=0.4$. Then $\Psi_{1}=0.22<$
$\widetilde{\Psi}_{1}=1.11$ and $\Psi_{2}=0.1>\widetilde{\Psi}_{2}=0.03$. It follows from (B) in Theorem 4 that $y_{1}$ goes to extinction and

$$
\begin{aligned}
\lim _{t \rightarrow+\infty} t^{-1} \int_{0}^{t} y_{2}(s) d s & =\frac{b_{2}-0.5 \sigma_{2}^{2}-\eta_{2}}{a_{22}}=\frac{0.41}{0.7} \\
& =0.59
\end{aligned}
$$

See Figure 1(c). In Case 4, we let $\gamma_{1}(u)=-0.03, \gamma_{2}(u)=0.01$, $\sigma_{1}^{2}=0.2$, and $\sigma_{2}^{2}=0.4$, that is, $\Psi_{1}=0.22>\widetilde{\Psi}_{1}=-0.22$ and 
$\Psi_{2}=0.1>\widetilde{\Psi}_{2}=0.095$. According to (C) in Theorem 4, we obtain

$$
\begin{aligned}
& \lim _{t \rightarrow+\infty} t^{-1} \int_{0}^{t} y_{1}(s) d s=\frac{\Psi_{1}-\widetilde{\Psi}_{1}}{\Psi}=1.22, \\
& \lim _{t \rightarrow+\infty} t^{-1} \int_{0}^{t} y_{2}(s) d s=\frac{\Psi_{2}-\widetilde{\Psi}_{2}}{\Psi}=0.0139 .
\end{aligned}
$$

Figure 1(d) validates this.

\section{Conclusions and Remarks}

This paper investigates a stochastic competitive system with distributed delay and general Lévy jumps. Under the assumption $\Psi>0$, the almost complete parameter analysis is fulfilled in detail. Our results imply that the general Lévy jumps can significantly change the properties of population models.

Some interesting and significant topics deserve our further engagement. One may put forward a more realistic and sophisticated model to integrate the colored noise into the model $[10,11,34]$. Another significant problem is devoted to stochastic model with infinite delays and general Lévy jumps. We will leave these for future investigation.

It should also be mentioned that "stability in time average" is not a good definition of persistence for stochastic population models. Some papers have introduced more appropriate definitions of permanence for stochastic population models, that is, stochastically persistent in probability or stochastic permanence (see, e.g., [35-37]). We will research these kinds of permanence of model (3) in detail in our following study.

\section{Competing Interests}

The authors declare that they have no competing interests.

\section{Acknowledgments}

This work is supported by the National Natural Science Foundation of China (no. 11501150), a Project of Shandong Province Higher Educational Science and Technology Program of China (J16LI09), the National Social Science Foundation of China (16BJL087), and the instructional reform item of Higher Education of Shandong Province (2015M091).

\section{References}

[1] Y. Kuang and H. L. Smith, "Global stability for infinite delay Lotka-Volterra type systems," Journal of Differential Equations, vol. 103, no. 2, pp. 221-246, 1993.

[2] T. Faria, "Asymptotic stability for delayed logistic type equations," Mathematical and Computer Modelling, vol. 43, no. 3-4, pp. 433-445, 2006.

[3] H. I. Freedman and J. H. Wu, "Periodic solutions of singlespecies models with periodic delay," SIAM Journal on Mathematical Analysis, vol. 23, no. 3, pp. 689-701, 1992.

[4] H. Bereketoglu and I. Győri, "Global asymptotic stability in a nonautonomous Lotka-Volterra type system with infinite delay," Journal of Mathematical Analysis and Applications, vol. 210, no. 1, pp. 279-291, 1997.
[5] K. Gopalsamy, Stability and Oscillations in Delay Differential Equations of Population Dynamics, vol. 74 of Mathematics and Its Applications, Kluwer Academic, Dordrecht, Netherlands, 1992.

[6] Y. Kuang, Delay Differential Equations with Applications in Population Dynamics, Academic Press, Boston, Mass, USA, 1993.

[7] R. M. May, Stability and Complexity in Model Ecosystems, Princeton University Press, Princeton, NJ, USA, 2001.

[8] X. Mao, G. Marion, and E. Renshaw, "Environmental Brownian noise suppresses explosions in population dynamics," Stochastic Processes and their Applications, vol. 97, no. 1, pp. 95-110, 2002.

[9] L. Zu, D. Jiang, D. O'Regan, and B. Ge, "Periodic solution for a non-autonomous Lotka-Volterra predator-prey model with random perturbation," Journal of Mathematical Analysis and Applications, vol. 430, no. 1, pp. 428-437, 2015.

[10] X. Li and X. Mao, "A note on almost sure asymptotic stability of neutral stochastic delay differential equations with Markovian switching," Automatica, vol. 48, no. 9, pp. 2329-2334, 2012.

[11] M. Liu and C. Bai, "Dynamics of a stochastic one-prey twopredator model with Lévy jumps," Applied Mathematics and Computation, vol. 284, pp. 308-321, 2016.

[12] W. Li, H. Song, Y. Qu, and K. Wang, "Global exponential stability for stochastic coupled systems on networks with Markovian switching," Systems \& Control Letters, vol. 62, no. 6, pp. 468-474, 2013.

[13] M. Liu and K. Wang, "A note on a delay Lotka-Volterra competitive system with random perturbations," Applied Mathematics Letters, vol. 26, no. 6, pp. 589-594, 2013.

[14] C. Lu and X. Ding, "Persistence and extinction in general non-autonomous logistic model with delays and stochastic perturbation," Applied Mathematics and Computation, vol. 229, pp. 1-15, 2014.

[15] J. Bao, X. Mao, G. Yin, and C. Yuan, "Competitive LotkaVolterra population dynamics with jumps," Nonlinear Analysis: Theory, Methods \& Applications, vol. 74, no. 17, pp. 6601-6616, 2011.

[16] J. Bao and C. Yuan, "Stochastic population dynamics driven by Lévy noise," Journal of Mathematical Analysis and Applications, vol. 391, no. 2, pp. 363-375, 2012.

[17] M. Liu and K. Wang, "Dynamics of a Leslie-Gower Holling-type II predatorprey system with Lévy jumps," Nonlinear Analysis: Theory, Methods \& Applications, vol. 85, pp. 204-213, 2013.

[18] M. Liu and C. Bai, "Optimal harvesting of a stochastic mutualism model with Lévy jumps," Applied Mathematics and Computation, vol. 276, pp. 301-309, 2016.

[19] Q. Ma, D. Ding, and X. Ding, "Mean-square dissipativity of several numerical methods for stochastic differential equations with jumps," Applied Numerical Mathematics, vol. 82, pp. 44-50, 2014.

[20] X. Zou and K. Wang, "Numerical simulations and modeling for stochastic biological systems with jumps," Communications in Nonlinear Science and Numerical Simulation, vol. 19, no. 5, pp. 1557-1568, 2014.

[21] R. Wu, X. Zou, and K. Wang, "Asymptotic properties of stochastic hybrid Gilpin-Ayala system with jumps," Applied Mathematics and Computation, vol. 249, pp. 53-66, 2014.

[22] X. Zhang and K. Wang, "Stability analysis of a stochastic Gilpin-Ayala model driven by Lévy noise," Communications in Nonlinear Science and Numerical Simulation, vol. 19, no. 5, pp. 1391-1399, 2014. 
[23] M. Liu, K. Wang, and Q. Wu, "Survival analysis of stochastic competitive models in a polluted environment and stochastic competitive exclusion principle," Bulletin of Mathematical Biology, vol. 73, no. 9, pp. 1969-2012, 2011.

[24] B. Øksendal and A. Sulem, Applied Stochastic Control of Jump Diffusions, Springer, Berlin, Germany, 2005.

[25] N. Ikeda and S. Watanabe, Stochastic Differential Equations and Diffusion Processes, North-Holland, Amsterdam, The Netherlands, 1981.

[26] N. Jacob, Y. Wang, and C. Yuan, "Stochastic differential delay equations with jumps, under nonlinear growth condition," Stochastics, vol. 81, no. 6, pp. 571-588, 2009.

[27] J. Bao and C. Yuan, "Comparison theorem for stochastic differential delay equations with jumps," Acta Applicandae Mathematicae, vol. 116, no. 2, pp. 119-132, 2011.

[28] C. Yuan and X. Mao, "Stability of stochastic delay hybrid systems with jumps," European Journal of Control, vol. 16, no. 6, pp. 595-608, 2010.

[29] Q. Han, D. Jiang, and C. Ji, "Analysis of a delayed stochastic predator-prey model in a polluted environment," Applied Mathematical Modelling, vol. 38, no. 13, pp. 3067-3080, 2014.

[30] H. Kunita, "Itô's stochastic calculus: its surprising power for applications," Stochastic Processes and Their Applications, vol. 120 , no. 5, pp. 622-652, 2010.

[31] R. S. Liptser, "A strong law of large numbers for local martingales," Stochastics, vol. 3, no. 3, pp. 217-228, 1980.

[32] Q. Ma, D. Ding, and X. Ding, "Mean-square dissipativity of several numerical methods for stochastic differential equations with jumps," Applied Numerical Mathematics. An IMACS Journal, vol. 82, pp. 44-50, 2014.

[33] X. Ding, Q. Ma, and L. Zhang, "Convergence and stability of the split-step $\theta$-method for stochastic differential equations," Computers \& Mathematics with Applications, vol. 60, no. 5, pp. 1310-1321, 2010.

[34] X. Li and G. Yin, "Logistic models with regime switching: permanence and ergodicity," Journal of Mathematical Analysis and Applications, vol. 441, no. 2, pp. 593-611, 2016.

[35] S. J. Schreiber, M. Benaïm, and K. A. S. Atchadé, "Persistence in fluctuating environments," Journal of Mathematical Biology, vol. 62, no. 5, pp. 655-683, 2011.

[36] M. Liu and C. Bai, "Analysis of a stochastic tri-trophic foodchain model with harvesting," Journal of Mathematical Biology, vol. 73, no. 3, pp. 597-625, 2016.

[37] M. Liu and M. Fan, "Permanence of stochastic Lotka-Volterra systems," Journal of Nonlinear Science, 2016. 


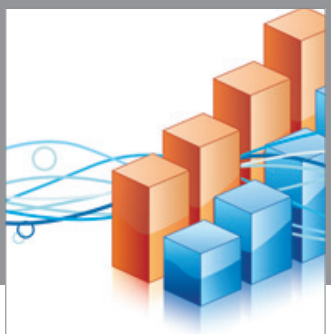

Advances in

Operations Research

vatem alat4

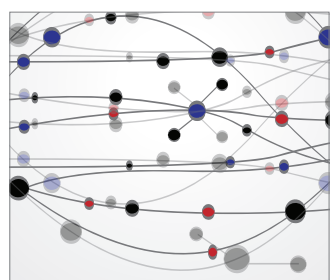

\section{The Scientific} World Journal
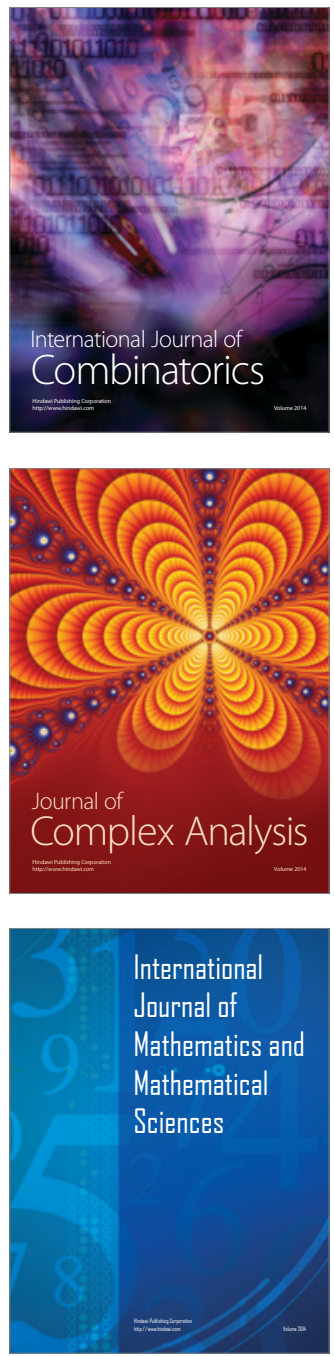
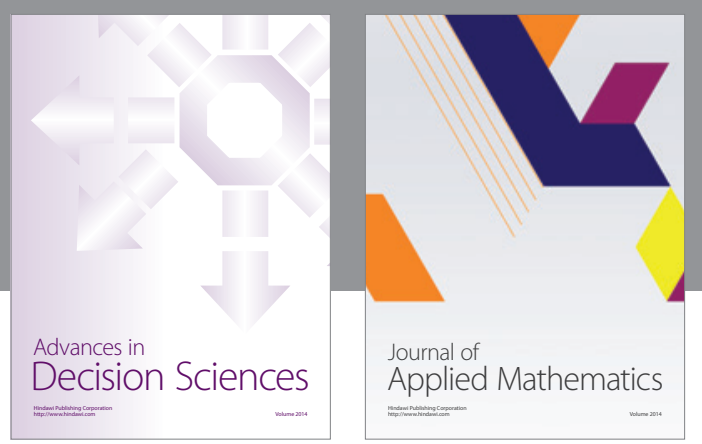

Algebra

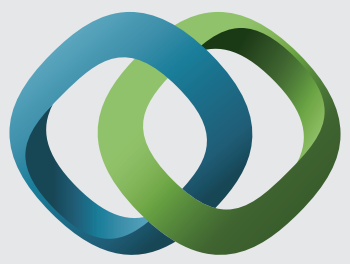

\section{Hindawi}

Submit your manuscripts at

http://www.hindawi.com
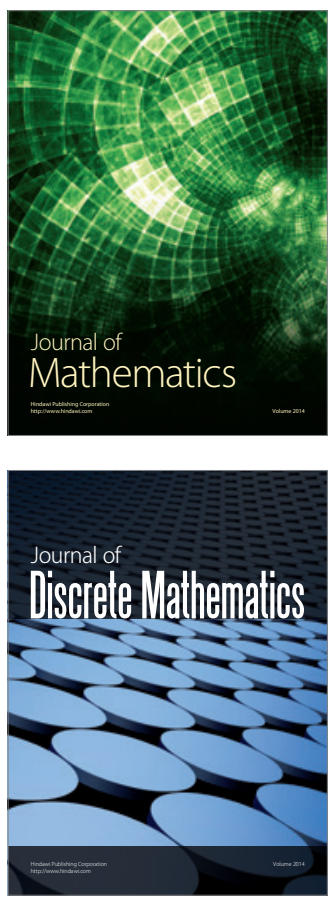

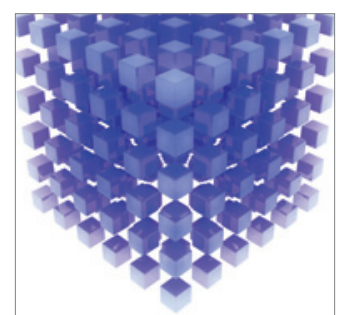

Mathematical Problems in Engineering
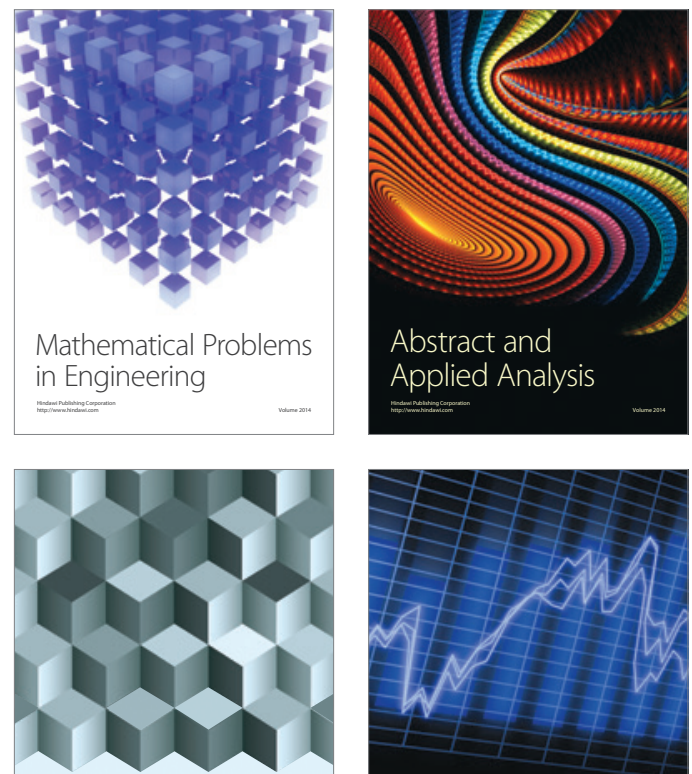

Journal of

Function Spaces

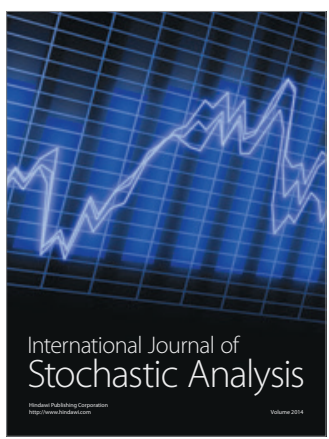

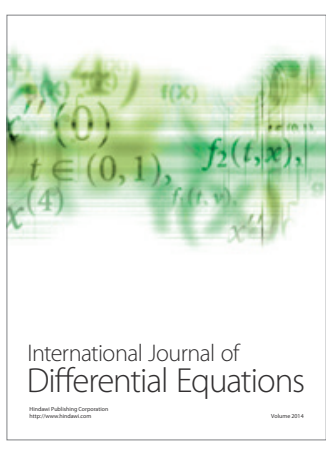
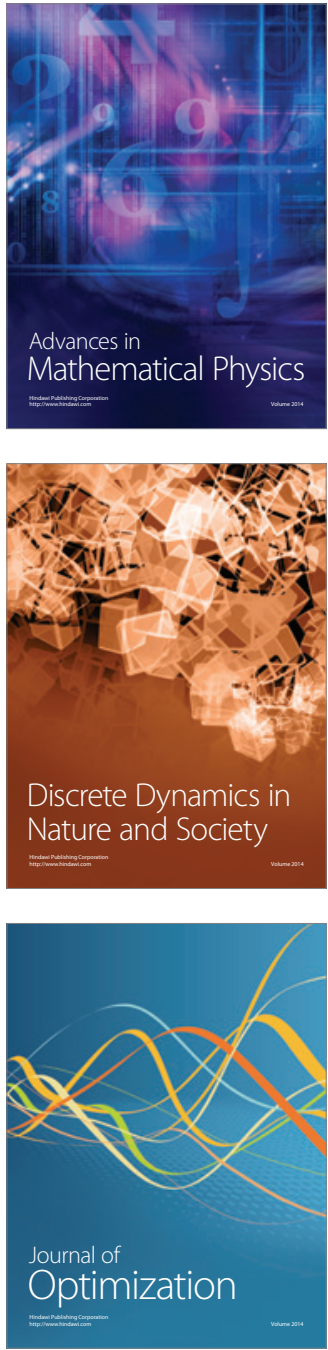